\title{
ILCEA
}

Revue de l'Institut des langues et cultures

d'Europe, Amérique, Afrique, Asie et Australie

27 | 2016

Approches ergonomiques des pratiques

professionnelles et des formations des traducteurs

\section{Le portail terminologique : un outil moderne de partage des connaissances}

Terminology Portals Provide a Modern Approach to Knowledge Sharing

\section{Caroline Champsaur et Michel Rochard}

\section{(2) OpenEdition}

\section{Journals}

Édition électronique

URL : http://journals.openedition.org/ilcea/4017

DOI : 10.4000/ilcea.4017

ISSN : 2101-0609

Éditeur

UGA Éditions/Université Grenoble Alpes

Édition imprimée

ISBN : 978-2-84310-336-0

ISSN : 1639-6073

Référence électronique

Caroline Champsaur et Michel Rochard, « Le portail terminologique : un outil moderne de partage des connaissances », ILCEA [En ligne], 27 | 2016, mis en ligne le 08 novembre 2016, consulté le 01 mai 2019. URL : http://journals.openedition.org/ilcea/4017 ; DOI : 10.4000/ilcea.4017

Ce document a été généré automatiquement le 1 mai 2019

(C) ILCEA 


\title{
Le portail terminologique : un outil moderne de partage des connaissances
}

\author{
Terminology Portals Provide a Modern Approach to Knowledge Sharing
}

Caroline Champsaur et Michel Rochard

1 Comme tant d'autres domaines, le champ terminologique n'échappe pas à l'influence grandissante des évolutions technologiques et de la problématique de la gestion de la connaissance et de l'information. Ces mutations nous amènent à revoir les théories, les méthodes, les pratiques et les outils et, de fait, à repenser les modèles.

2 Travaillant dans un organisme international de recherche chargé notamment de produire des analyses et des préconisations sur ces mutations, les traducteurs de l'Organisation de coopération et de développement économiques (OCDE) qui produisent et gèrent de la terminologie au quotidien ont entrepris une réflexion consciente sur ces évolutions afin de bâtir un modèle d'ergonomie terminologique fondé sur le partage de connaissances entre traducteurs et experts. Ce modèle s'est matérialisé fin 2012 sous la forme d'un portail terminologique baptisé AGORA. Le présent article est consacré au cheminement de cette réflexion et aux solutions auxquelles elle a abouti.

3 Depuis une cinquantaine d'années, les traducteurs de l'OCDE consacrent une partie de leur activité à la terminologie. Derrière cette continuité apparente, ce travail a connu des évolutions fondamentales d'ordre technologique bien évidemment, mais aussi et surtout d'ordre méthodologique.

4 Nous avons d'emblée indiqué que la production terminologique de la Division de la traduction de l'OCDE était le fait de traducteurs et c'est important, car notre approche terminologique originelle a été guidée par la recherche de solutions de traduction. En effet, le premier souci des traducteurs dans ce domaine est de consigner des solutions opérantes pour la traduction de termes spécialisés rencontrés au cours de leur activité, afin de ne pas «réinventer l'eau chaude ${ }^{1}$ ». Ce travail a donc consisté à apparier des termes (en l'occurrence anglais) et une ou plusieurs solutions de traduction (en français). 
Comme l'OCDE est un organisme de réflexion qui couvre de très nombreux domaines, ce travail a été enrichi par l'indication de ces domaines. À titre d'exemple, le terme anglais " consolidation" peut relever de la science des sols en agriculture ou en environnement auquel cas, on parlera de " tassement » d'un sol, mais aussi être utilisé dans le monde de la finance où il renverra à l'idée de "concentration » du capital dans un secteur dans le cadre de fusions d'entreprises, ou encore en comptabilité où la « consolidation » désigne la réunion en un même ensemble les états comptables des composantes d'un groupe multinational.

Cependant, l'indication du domaine ne suffisait pas. La complexité croissante des textes à traduire et de la logique qui les sous-tend a poussé les traducteurs à faire évoluer leur travail en accordant de plus en plus de place aux définitions des termes traités. Or, non seulement cette évolution est conforme à celle de la démarche terminologique, mais encore, elle est professionnellement indispensable.

\section{Auteur-expert et traducteur, deux travailleurs de la connaissance complémentaires}

7 Première étape, la conception du message, lors de laquelle l'auteur-expert utilise sa connaissance de son domaine et ses capacités cognitives pour rédiger ce message. Dans son choix de formulation, l'auteur-expert effectue naturellement un choix terminologique pour sélectionner le terme rendant compte de sa réflexion.

Seconde étape, la traduction de ce message, dont l'objectif est de produire une solution de traduction «logiquement et fonctionnellement équivalente » à l'original de l'expert (Rochard, 2006: 104). Pour ce faire, le traducteur utilise sa propre connaissance du domaine, ses capacités cognitives ainsi que tous les éléments d'information linguistiques ou non contenus dans le message de l'expert. Ce message est souvent complexe, mais on sait que la "traduction est par définition une activité intellectuelle complexe de résolution de problèmes » (Fougner Rydning, $2008: 1$ ). Pour surmonter ces problèmes et cette complexité, la démarche du traducteur consiste à entamer une enquête logique, telle que décrite par le philosophe pragmatique américain John Dewey (Deledalle, 1995 ; Dewey, 1938). Le traducteur cherche à comprendre quel concept ou découpage du monde correspond aux mots de l'expert. C'est la « rétroconception du message » (Rochard, 2006 : 112).

9 Pour le traducteur, la connaissance tacite (Kimble, 2013) que l'auteur-expert a de son domaine serait très utile, car elle lui permettrait notamment de lever ses incertitudes quant à la compréhension du message. Mais le caractère intangible de la connaissance tacite fait obstacle à cette appropriation directe de la connaissance de l'expert. Le traducteur doit donc contourner cette difficulté en orientant son enquête sur l'identification des marqueurs plus explicites du savoir de l'expert. Nous en retiendrons quatre :

1. Les marqueurs logiques. Ce sont des éléments fondamentaux de l'enquête qui relèvent de l'analyse du contexte non linguistique dans la Théorie interprétative de la traduction (Seleskovitch \& Lederer, 1989). Néanmoins, les marqueurs logiques sont beaucoup moins présents dans les textes rédigés en anglais que dans les textes en français ;

2. Le nom de l'expert, ou le ou les réseaux d'experts auxquels il appartient. Le contacter est un excellent moyen d'obtenir une explicitation du message et de lever les incertitudes. C'est 
malheureusement un levier difficilement mobilisable en raison du temps dont dispose l'expert ou ses collègues pour aider le traducteur ${ }^{2}$;

3. Les publications de l'expert ou de ses réseaux. On y a certainement là des éléments de connaissance explicite très précieux. Malheureusement, la contrainte de temps s'exerce dans ce cas sur le traducteur qui ne peut se permettre d'éplucher ces sources dans le temps qui lui est imparti ${ }^{3}$;

4. Les termes spécialisés. Ce sont des marqueurs de connaissance. Partant de ces marqueurs, le travail terminologique va précisément viser à expliciter cette connaissance à l'intention du lecteur ou du traducteur, le moyen privilégié d'explicitation étant la définition de concept. Plus précisément, il s'agit d'une définition terminologique qui est celle du créateur du concept ou à tout le moins d'un expert du domaine spécialisé dans lequel est utilisé le terme (de Bessé, 1990).

Les informations reflétant les trois derniers marqueurs peuvent servir à une réutilisation ultérieure de la connaissance :

- Les liens entre l'auteur-expert et les autres experts de son domaine ou de domaines connexes sont identifiables au travers de communautés d'experts (Wenger, 1999). Hustad et Bechina parlent quant à elles de « réseaux distribués de connaissance " ( distributed network of knowledge - DNoOK») qu'elles définissent comme :

[...] a flexible group of professionals and experts operating in a geographically dispersed context, sharing common interests and experiences related to business topics, using a suitable context (' $b a$ ') for their knowledge activities, thereby building a common store of knowledge aiming to achieve learning and innovation. (Hustad \& Bechina, 2012 : 123)

- Pour les publications, il est également possible de consulter des bases de références bibliographiques. Créées depuis le début des années 1960, elles étaient à l'origine fréquemment nommées «banques de données» et définies dans le Journal officiel comme un « ensemble de données relatif à un domaine défini de connaissances et organisé pour être offert aux consultations d'utilisateurs » (JORF, 1982). Depuis le début des années 1990, le terme «bibliothèque numérique » regroupant des collections numériques motivées par une politique documentaire définie s'est imposé. Ces contenus, organisés pour faciliter la recherche et la consultation, sont soit des documents papiers qui ont été numérisés, soit des documents nativement numériques (ENSSIB, 2013).

- Les termes spécialisés, quant à eux, sont stockés dans des bases de données, banques ou portails terminologiques.

11 Le traducteur peut naturellement utiliser les références existantes (réseaux d'experts, bibliothèques numériques ou bases de données terminologiques). Mais, comme nous l'avons noté en introduction, en ce qui concerne la terminologie, le stockage des informations terminologiques glanées ici ou là et la conservation de ses solutions de traduction sont pour le traducteur une activité naturelle, inhérente à sa pratique professionnelle. Cette activité est sans doute un des vecteurs les plus efficients d'accès à la connaissance de l'expert ; en ce sens, le travail terminologique tel qu'il est pratiqué par les traducteurs de l'OCDE est bien un travail de gestion de la connaissance.

12 On est dès lors en droit de se demander si le traducteur peut se passer du concours de l'auteur-expert pour son travail terminologique. C'est en effet ce qui s'est produit pendant plus de vingt années de travaux terminologiques à l'OCDE. Tandis que les experts de l'Organisation introduisaient des définitions de concepts, voire des glossaires, dans certains grands textes de l'OCDE, les traducteurs, comme leurs homologues d'autres organisations internationales, concentraient leur travail terminologique sur la recherche et la consignation de solutions de traduction de termes. En fait, la seule rencontre entre 
ces deux activités intervenait lors de la traduction des glossaires contenus dans les publications des différentes directions de l'OCDE par la Division de la traduction, et les traducteurs ne retenaient dans leurs propres glossaires que les solutions de traduction mises au point dans le cadre de ces exercices. Ces vingt dernières années, devant la complexité et l'interdisciplinarité des textes à traiter, les traducteurs ont pris conscience que les solutions de traduction ne leur suffisaient plus et qu'ils avaient besoin d'accéder à la connaissance des experts. C'est à partir de ce moment qu'ils ont enrichi leurs propres glossaires par des définitions de concepts rencontrées au cours de leurs traductions ou figurant dans les publications de l'Organisation.

Pour ce faire, les traducteurs spécialisés disposent de deux méthodes ; d'une part, la veille terminologique qui consiste à repérer et consigner toute définition de concept figurant dans les textes qu'ils traduisent; d'autre part, le dépouillement systématique des publications des experts de leur domaine spécialisé ou des réseaux constitués par ces experts. Ces stratégies se heurtent cependant à plusieurs obstacles.

Dans le cas de la veille terminologique à partir des textes à traduire, le principal obstacle réside dans le fait que les textes de l'OCDE - documents internes ou publications - sont loin d'être systématiquement traduits, de sorte qu'une partie non négligeable de la production terminologique des experts échappe aux traducteurs. Autrement dit, le problème tient au fait que la chaîne de production et de diffusion des documents et publications ne passe pas nécessairement par la traduction. Il faut donc développer une forme de collaboration plus institutionnelle et plus continue avec les experts pour mener à bien cette veille terminologique et améliorer ainsi les connaissances spécialisées des traducteurs et la qualité de leurs travaux.

Pour ce qui concerne le dépouillement de la littérature des experts ou de leurs réseaux, le premier obstacle réside dans le caractère chronophage de cette démarche. Ensuite, cette approche est entravée par la difficulté d'identifier le véritable champ de compétence de l'expert à un moment $t$. Ainsi, un expert ayant l'étiquette générale « agriculture » sera peut-être en réalité un expert des indicateurs agro-environnementaux. Un de ses collègues sera quant à lui spécialiste des échanges agro-alimentaires. L'identification des réseaux de ces experts est encore plus difficile. En outre, la population d'experts est extrêmement mouvante, ne serait-ce que du fait des règles internes de mobilité des agents de l'Organisation. Un expert de l'environnement peut fort bien demander sa mutation pour la Direction de la science, de la technologie et de l'innovation dans le cadre d'un projet correspondant à son profil initial.

Il s'agit là de contraintes pesant lourdement sur la collecte de définitions de concepts en vue de leur intégration dans une terminologie fondée sur la connaissance. Mais la difficulté ne s'arrête pas là. Au-delà de la collecte, il y a la question de la gestion de la connaissance.

17 En effet, les concepts sur lesquels s'appuient les activités terminologiques des traducteurs ne sont pas statiques. Ainsi, le Modèle de convention fiscale de l'OCDE concernant le revenu et la fortune, publié pour la première fois en 1977, a toujours contenu des définitions de concepts établies à l'origine à des fins de certitudes juridiques pour l'application du modèle. La version de 1977 de ce modèle correspondait à une époque où les affaires fiscales internationales étaient relativement simples. Aujourd'hui, nous sommes à l'ère de l'économie numérique et de la possibilité de déplacer instantanément des revenus par des moyens électroniques. Nul doute que certains concepts seront appelés à être redéfinis et que le sens de nouveaux concepts devra être explicité au fil de ces évolutions. Or, les 
traducteurs spécialisés n'auront pas été associés aux discussions sur ces évolutions qui auront débouché sur une redéfinition de ces concepts. Là encore, l'idéal est de pouvoir établir une collaboration entre les traducteurs et les experts en vue d'une gestion active de la connaissance.

De telles collaborations sont possibles. Toujours dans le domaine de la fiscalité internationale, un expert des prix de transfert ${ }^{4}$ a ainsi mis à jour les définitions d'une cinquantaine de termes consacrés à ce thème qui figureraient dans les ressources terminologiques de la Division de la traduction.

Il apparaît donc que l'optimisation du travail terminologique des traducteurs passe sans doute par une sensibilisation des experts à l'existence et à l'utilité pour eux de ce travail et, in fine, à une collaboration par des moyens efficients.

De fait, l'auteur-expert a lui aussi intérêt à l'existence d'un tel travail terminologique :

- En donnant la(les) définition(s) des concepts qu'il emploie, l'auteur-expert transforme sa connaissance tacite en connaissance explicite, la mettant ainsi à la disposition des autres. Ses travaux sont ainsi plus lisibles et sa pensée mieux comprise. Dans un monde de surabondance d'informations, la clarté des messages est une valeur clef : elle améliore la diffusion des contenus et l'impact de ces messages.

- L'ajout de propositions de traductions par les traducteurs permet en sus d'élargir la diffusion des concepts employés par l'auteur-expert. Le message a un meilleur coefficient de pénétration dans le public visé, touchant des personnes de langues maternelles différentes.

21 Au-delà de ces effets bénéfiques sur la diffusion et sur la clarté du message de l'auteurexpert, la construction et l'existence de bases de données ou de portails terminologiques spécialisés présentent plusieurs avantages d'ordre organisationnel.

Il est en effet fréquent que des experts travaillant pour un même projet de recherche s'accordent sur les termes spécialisés récurrents qu'ils vont utiliser depuis l'écriture d'un rapport jusqu'à sa publication. Ce premier travail terminologique va être un facteur de cohérence de l'ensemble du projet.

De même, s'ils ont élaboré entre eux un glossaire spécialisé et l'ont transmis aux traducteurs susceptibles de prendre en charge leur rapport, les experts apporteront un gain d'efficience au processus de traduction en mettant à la disposition des traducteurs les éléments de connaissance qui faciliteront leur travail d'enquête en affinant leur compréhension du message.

De plus, comme on l'a vu, tous les organismes de recherche connaissent un renouvellement de leurs effectifs et la stabilisation de la connaissance des experts dans des glossaires spécialisés est un facteur de transmission intergénérationnelle de la connaissance au sein de l'organisation.

Enfin, dans des organisations internationales où les experts viennent de pays des plus divers avec des cultures institutionnelles différentes, la fixation de terminologies spécialisées propres à l'organisation est un facteur de dépassement de ces différences et d'intégration des nouveaux venus.

Pour illustrer l'intérêt d'une telle démarche terminologique collaborative pour les experts, nous retiendrons le projet de rédaction et de traduction de la réédition du Manuel de Frascati (OCDE, 2002). Les rédacteurs de cette nouvelle édition ont prévu d'introduire en annexe de cette publication un glossaire définissant une centaine de termes importants afin d'en éclairer le sens pour les lecteurs. Sachant que cette publication 
serait traduite en français par la Division de la traduction et que cette dernière gère des ressources terminologiques, les rédacteurs se sont adressés aux traducteurs pour mettre au point une méthode de collaboration. Ils se sont efforcés de transmettre le glossaire le plus en amont possible, de façon que les traducteurs engagés dans ce projet puissent s'entendre en amont également sur la traduction des principaux termes employés dans le texte et donc d'assurer une meilleure cohérence de la traduction.

Mais ce n'est pas tout, car la terminologie utilisée dans ce Manuel sera en partie reprise dans la nouvelle édition du Manuel d'Oslo (OCDE, Commission européenne, 2005) qui devrait paraître, toujours en anglais et français, en 2017 et sans doute même dans la nouvelle édition du Système de comptabilité nationale (ONU et al., 1993). La question grandissante de la mesure des activités de recherche-développement et/ou de l'innovation est en effet abordée dans ces trois documents normatifs. Le glossaire du Manuel de Frascati est donc particulièrement intéressant pour les experts qui s'assurent d'une meilleure cohérence terminologique de ces trois documents ainsi que de la cohérence de leur traduction. Qui plus est, en confiant la gestion de cette terminologie aux traducteurs, ceux-ci seront certains d'une continuité de l'utilisation et de la transmission de leurs connaissances quel que soit leur parcours professionnel ultérieur. Qui sait en effet combien d'experts ayant travaillé sur le Manuel de Frascati seront encore associés à l'élaboration du Système de comptabilité nationale dans quelques années?

Auteur-expert et traducteur ont tous deux intérêt à contribuer au travail terminologique : facteur d'efficience méthodologique pour tous les deux dans un premier temps, il permet dans un deuxième temps d'élargir la portée du message et au final, de pallier la perte de connaissances. Le traducteur ne saurait donc se contenter d'être un simple consommateur (Rochard, 1992). Comme l'auteur-expert, il est lui aussi un travailleur de la connaissance dont la contribution vient compléter celle de l'expert.

Comment les contributions de l'auteur-expert et du traducteur peuvent-elles se compléter? On sait que la notion de définition est loin d'être univoque (Blanchon, 1997 : 170). Il convient donc de s'arrêter sur les apports respectifs de l'auteur-expert et du traducteur au portail de connaissances.

\section{Auteurs-experts et traducteurs : quels apports terminologiques?}

Dans le cadre de la rédaction d'un ouvrage, les auteurs-experts peuvent, comme on l'a vu, être amenés à rédiger des définitions. Ces définitions ont une fonction très particulière : faciliter la compréhension par le lecteur des termes techniques employés dans l'ouvrage. Les auteurs-experts doivent tenter de cerner le lectorat intéressé par leur ouvrage et adapter la rédaction de leurs définitions pour ce public. Comme les lexicographes, ils :

[...] doivent adapter la paraphrase synonymique scientifique afin de la transcoder dans une forme écrite capable d'être reçue par le groupe socio-culturel qu'ils ont défini comme étant le récepteur potentiel de l'information contenue dans le dictionnaire. (Blanchon, 1997 : 71)

31 Les auteurs-experts peuvent ainsi être amenés à rédiger deux définitions distinctes pour un même terme: une définition dite d'expert et une définition simplifiée. Deux définitions sont ainsi proposées pour le terme « capital loss » :

Définition d'expert : 
Capital gains or losses are increases or decreases in the value of the assets of institutional and other units which are due to changes in market prices, discovery of new mineral deposits and other natural resources, natural growth of timber, depletion mineral deposits, unforeseen obsolescence, theft, major catastrophes and other events except the purchase and sale of assets, the normal wear and tear, accidental damage, losses in tangible assets, the writing off of bad debts and other flows which are recorded in the transaction accounts. ( OECD/Economics Department/Economics Glossary, 2005) ${ }^{5}$

Définition simplifiée :

The loss from the sale of a capital asset. (OECD/Glossary of Tax Terms/OECD Website, last accessed on 13-04-2015)

La définition d'expert de "capital loss » est une définition descriptive non prescriptive. Elle décrit un événement possible pour un capital. La définition de cet événement vise à en délimiter précisément le sens en :

1. opposant le terme à son antonyme (capital gain) et en incluant les deux concepts dans un système plus général des événements influant sur les modifications possibles du capital. La définition apparaît à la fois comme distinctive et englobante.

2. précisant les facteurs contribuant à l'émergence de cet événement (« which are due to changes in market prices, discovery [...]»). La définition cherche à donner la liste exhaustive de ces facteurs.

Cette définition d'expert vise donc la précision et l'exactitude, voire l'exhaustivité. La définition simplifiée de "capital loss» est elle aussi une définition descriptive non prescriptive. Plus courte, elle vise à donner le sens grossier du concept sans le replacer dans un système ni lister les facteurs sous-jacents. Outre les explications sur le sens du terme (la définition), le traducteur recherche d'autres informations de nature langagière : des propositions de traduction en langue cible, d'éventuelles précisions d'emploi de ces traductions et des précisions sur le terme original. Ce sont ces informations qu'il consignera utilement dans la fiche terminologique. d'utilisation. Le traducteur peut ainsi préciser que le terme original s'emploie dans un pays particulier. Le terme "alfalfa » utilisé au Canada anglophone et aux États-Unis est remplacé par le terme "lucerne » en Australie, en Nouvelle-Zélande et au Royaume-Uni. Parfois, le contexte d'emploi est plus restreint et peut se limiter à une organisation ou une institution. On trouvera ainsi très souvent des indications du type «s'emploie à/ dans/pour... ». Le traducteur peut également souhaiter ajouter une information motivant la proposition de traduction. Pour le terme "global balancing premium ", le traducteur propose la traduction française «prime d'équilibre entre épargne et investissement au niveau mondial » en précisant :

[...] bien que l'expression anglaise mette l'accent plutôt sur l'équilibre mondial des paiements courants, la force économique en jeu porte sur l'équilibre mondial entre épargne et investissement, et il semble plus judicieux d'insister sur ce dernier point dans la traduction française. En fin de compte, il s'agit d'une prime qui sert à ajuster les taux d'intérêt réels afin d'assurer l'égalité comptable entre épargne et investissement au niveau mondial ${ }^{7}$.

Dans les précisions d'emploi des termes cibles, le traducteur pourra souligner qu'une des traductions proposées est à présent obsolète, ce qui lui permet de ne pas systématiquement privilégier le terme cible le plus fréquemment rencontré. Il peut également différencier des propositions de traduction par le contexte d'emploi au sein d'un même domaine. En agriculture par exemple, le terme anglais " auxiliary organism » 
peut se traduire par "agent de lutte biologique» ou "auxiliaire de lutte biologique (ALB) ». Même s'il peut s'appliquer aux invertébrés, le terme cible «agent de lutte biologique » sera privilégié pour les micro-organismes (bactéries, champignons, virus). La seconde proposition de traduction « auxiliaire de lutte biologique " s'emploie quant à elle uniquement pour les invertébrés (insectes). Les précisions d'emploi servent également à indiquer quelle forme linguistique (singulier, pluriel) est à éviter ou à privilégier.

L'information au niveau de la langue apportée par le traducteur est une valeur ajoutée qui complète à merveille la définition de l'expert. Auteur-expert et traducteur se complètent sans empiéter sur le terrain de l'autre, ils ont tout intérêt à collaborer.

\section{Le portail : un modèle ergonomique adapté à la collaboration terminologique}

l'ergonomie organisationnelle, tandis que les trois dernières relèvent de l'ergonomie cognitive qui porte sur «les processus mentaux au travail» (Lavault-olléon \& Carré, 2012 ; Lavault-Olléon, 2011).

\section{Ergonomie organisationnelle}

\section{Une collaboration dématérialisée}

Le portail permet de donner des rôles différents aux différents groupes d'utilisateurs. Une première catégorie d'utilisateurs sera ainsi uniquement autorisée à visualiser un ensemble défini d'entrées. Une seconde catégorie sera autorisée en sus à créer de nouvelles entrées et à commenter des entrées existantes. Outre ces rôles, une troisième catégorie sera également autorisée à modifier des entrées. Un utilisateur a également la possibilité d'être averti des nouvelles entrées, des commentaires, des modifications sur l'ensemble des entrées et sur celles qui relèvent d'un domaine défini. Ces fonctionnalités permettent ainsi de mettre en place une collaboration active sans contact direct: un 
auteur saisit une nouvelle définition, le traducteur en est averti, il propose une ou plusieurs traduction(s) qui sont notifiées à l'auteur, etc. Il est ainsi possible de collaborer en s'affranchissant des barrières géographiques et de créer des réseaux d'experts.

\section{Une collaboration désynchronisée}

\section{Ergonomie cognitive}

\section{La conservation du capital de connaissances}

Le manque de disponibilité des différentes parties prenantes est souvent une cause d'échec à la collaboration. L'utilisation d'un portail permet à chacun de collaborer lorsqu'il est disponible en s'affranchissant des réunions communes. La messagerie électronique a depuis longtemps détrôné le téléphone comme premier outil de communication dans les organisations en permettant un envoi de message quasiimmédiat sans obliger pour autant le destinataire à répondre dans l'instant. Le portail fonctionne d'une façon similaire : une entrée mise sur le portail est accessible à tous dans l'instant (instantanéité de la transmission d'information) à condition qu'elle ait le statut nécessaire mais l'approbateur peut différer sa validation.

\section{Une validation des données terminologiques}

et leur donnent un statut "final» ou "official» (pour les entrées enrichies par une définition de l'OCDE). Les traducteurs n'ont pas à vérifier si de nouvelles entrées ont été ajoutées : ils en sont avertis par un courriel hebdomadaire automatique leur indiquant la présence de nouvelles entrées dans leur domaine. Ils doivent alors valider ces entrées, c'est-à-dire les enrichir si besoin ou revoir leur format. À cette étape, l'approbateur envoie généralement un courriel au créateur pour l'informer des modifications apportées et les expliquer.

Enfin, dans AGORA, il existe des filtres de diffusion sur l'intranet des données terminologiques et donc des connaissances correspondantes en fonction de leur niveau de validation, trois statuts sur l'intranet : le statut « revised draft » qui peut être donné par l'auteur de l'entrée dès sa création et qui indique que l'entrée n'a pas encore été approuvée, et les statuts «final » et « official » qui ne sont donnés qu'après approbation. Dans le cadre d'une version accessible à un public potentiel extérieur à l'OCDE, ces statuts seraient ramenés à deux (final et official), ce public ne devant pas avoir accès à la phase d'organisation et de validation des données préparant la diffusion des connaissances.

Au sein d'une organisation ou d'une entreprise, les connaissances représentent une des ressources les plus précieuses. Le départ d'un expert s'accompagne malheureusement souvent de la perte de son savoir et de ses contacts avec des réseaux d'experts extérieurs à l'organisation ou l'entreprise. Le travail de transformation de la connaissance tacite en une connaissance explicite est un enjeu pour l'avenir, car il permet aux connaissances détenues par un expert d'être pérennisées sous forme explicite et utilisées même après son départ. À l'OCDE, le portail AGORA est l'outil institutionnel de gestion des connaissances terminologiques. D'autres outils sont utilisés pour d'autres pans de la connaissance (logiciel d'archivage pour les documents officiels par exemple). 


\section{La transmission des connaissances}

47 La formation de nouvelles personnes est, nous l'avons vu, un enjeu de taille pour toute organisation. Indépendamment de son niveau d'études, l'arrivée d'une nouvelle personne suppose qu'elle acquière un certain nombre de savoirs. Le portail est ainsi un outil précieux pour former les nouveaux auteurs-experts et les nouveaux traducteurs.

Le portail terminologique AGORA est naturellement très utile pour compléter la formation des traducteurs fraîchement arrivés. Il sert également aux experts qui, même lorsqu'ils partagent la même langue maternelle, l'utilisent pour parfaire leur connaissance du vocabulaire utilisé à l'OCDE. Ainsi, des experts fiscalistes d'Australie, des États-Unis ou du Royaume-Uni peuvent s'entendre sur le choix des termes à utiliser à l'OCDE et leur signification exacte.

\section{La réutilisation des connaissances}

La rédaction de glossaires est un travail fastidieux souvent négligé. Le stockage centralisé de termes et de leurs définitions permet de simplifier la création de glossaires (et ce, en plusieurs langues), simplifiant ainsi la lecture des ouvrages. Naturellement, cette aide à la lecture peut également se concrétiser sous la forme d'aides en ligne (infobulles, par exemple).

En plaçant la connaissance au cœur de sa conception, le portail est également en phase avec les grandes tendances de la terminologie moderne, car, comme le remarque Humbley :

Ce n'est pas un hasard si la plus grande manifestation européenne de terminologie est le congrès de la Société pour la terminologie et le transfert des connaissances [...]. (Humbley, 1998)

51 ou encore,

Depuis une quinzaine d'année, en revanche, [la terminologie...] s'oriente vers de nouvelles applications, en particulier celle de la gestion des connaissances. (Humbley, 2001) 
Les champs d'AGORA et leur référentiel théorique et méthodologique

Cet encadré vise à présenter plus en détail les principaux champs du portail AGORA du point de vue fonctionnel, mais aussi en rattachant ces descriptions à diverses références ergonomiques, méthodologiques et théoriques touchant à la terminologie. Les champs répertoriés ici sont uniquement des champs visibles par l'utilisateur du portail en lecture seule.

Terme

- Champ terminologique élémentaire. Dans AGORA, c'est le terme anglais qui constitue le principal repère terminologique. Il peut être assorti de variantes ou synonymes, tandis que les termes français représentent surtout des «traductions» du terme anglais traitées indifféremment.

- Dans AGORA, le terme est à la fois un élément linguistique de dénomination et un marqueur de connaissance spécialisée dans la mesure où il est lié au moins à un ensemble plus ou moins complet de balises (domaines, spécialités, mots-clés, pays ou institutions utilisant ce terme, ou autres précisions d'emploi) Dans le meilleur des cas, il est aussi relié à une ou plusieurs définitions d'experts ou notes techniques du créateur de l'entrée. La démarche et l'organisation d'AGORA à partir du terme est tout à fait conforme à l'idée «que les termes constituent le point de départ de la réflexion des spécialistes et des interrogations des traducteurs. » (Humbley, 2011)

- Du point de vue de l'ergonomie organisationnelle, le terme est un vecteur spontané et privilégié de recherche et donc de l'accès à la connaissance terminologique. En tant que tel, le terme se situe donc à l'interface de l'ergonomie organisationnelle et de l'ergonomie cognitive. (Lavault-Olléon, 2011)

- Dans la typologie des données terminologiques de Gouadec, le terme et ses variantes ou synonymes relèvent de la rubrique de dénomination. (Gouadec, 1990)

Définition

- Comme on l'a vu, les définitions d'AGORA se subdivisent en définitions d'experts établies par des spécialistes de l'Organisation et en définitions simples établies à des fins de vulgarisation ou d'information succincte. Toutes les définitions émanant de l'OCDE doivent être assorties d'une source et d'une date ou, en tant que de besoin, d'un lien hypertexte.

- Par son caractère central dans une entrée terminologique complète la définition peut être considérée comme le pivot de l'ergonomie cognitive d'AGORA.

- Les définitions d'AGORA relèvent des rubriques encyclopédiques. (Gouadec, 1990)

Note technique

- Dans AGORA, la note technique vient compléter la définition d'expert ou s'y substituer en cas de besoin. Historiquement, les notes techniques ont généralement été introduites en français dans une démarche de terminologie bilingue classique qui n'incluait pas la définition.

- La note technique procède donc de l'ergonomie cognitive d'AGORA.

- La note technique relève des rubriques encyclopédiques. (Gouadec, 1990) 
Champs de balisage (domaine, spécialité, mot-clé, pays, institution)

- Tous ces champs délimitent le contexte d'emploi d'un terme: grand domaine de connaissance, spécialité plus fine et transversale, référence à un projet spécifique de recherche entrepris par l'OCDE et indication du pays ou de l'institution utilisant ce terme en particulier dans le sens ressortant de la définition.

- De ce fait, ils ont aussi une fonction cognitive dans leur rapport avec la définition et ils peuvent aussi introduire une dimension cognitive minimale mais utile lorsque l'entrée terminologique ne comporte pas (encore) de définition. Ils sont donc une composante de l'ergonomie cognitive.

- Du point de vue de l'ergonomie organisationnelle, ils offrent de multiples voies d'accès au contenu terminologie, ce qui permet à l'utilisateur de s'approprier l'outil, conformément à la politique de la Division de la traduction de l'OCDE. (Champsaur \& Rochard, 2010)

- Ces champs relèvent ainsi tout à la fois des rubriques délimitatives, des rubriques de gestion ou plus précisément d'indexation, et des rubriques encyclopédiques. (Gouadec, 1990)

Champs de traduction

- AGORA comporte deux champs de traduction. D'une part la traduction, champ répétable qui permet de dresser une liste de solutions de traduction dans un contexte délimité par les champs de balisage. D'autre part, un champ plus rarement rempli qui apporte une précision sur l'emploi d'une traduction dans un contexte délimité, par contraste avec d'autres solutions de traduction du terme original (voir exemples dans la section «Auteurs-experts et traducteurs : quels apports terminologiques?»). La précision peut avoir aussi une fonction de mise en garde contre des solutions de traduction à éviter.

- Dans la terminologie multilingue classique, ces champs ont une valeur descriptive pour les traductions et délimitative pour la précision de traduction. La contribution des organisations internationales au développement de la terminologie multilingue est soulignée par Raus (2013).

- Ces deux catégories de champs s'inscrivent donc dans une ergonomie cognitive restreinte.

- Les traductions relèvent de rubriques linguistiques, mais aussi cognitives, tandis que la précision est une rubrique qui peut être à la fois de délimitation et de protection. (Gouadec, 1990)

Champ de statut

- Dans AGORA, l'entrée telle que la visualise l'utilisateur peut relever de deux statuts de validation. Le statut «final » renvoie à une entrée validée par un administrateur spécialisé du portail, mais qui ne comporte pas de définition provenant de l'OCDE. Les autres entrées ont un statut « officiel » qui signale qu'elles comportent une définition établie par l'OCDE.

- Le champ de statut intervient dans l'ergonomie organisationnelle d'AGORA au sens où il permet de définir ce que l'on veut afficher ou non selon les différentes catégories d'utilisateurs. Mais il a aussi une fonction d'indication de la validité/fiabilité et du contenu cognitif de l'entrée, ce qui relève de l'ergonomie cognitive du portail.

- Le champ de statut relève des rubriques de gestion et de validation. (Gouadec, 1990)

\section{AGORA : l'alliance de la gestion de la connaissance et de la proposition de solutions de traduction}

Il est d'usage de distinguer différents types d'approches terminologiques : la terminologie conceptuelle, textuelle, descriptive, prescriptive ou encore normative. On signalera à cet 
égard la parution prochaine d'une contribution au débat portant sur la théorie du concept en terminologie (Roche, à paraître). Là où les entrées descriptives cherchent à rendre compte du sens d'un concept et de l'usage d'un terme, les entrées prescriptives visent à délimiter les contours exacts du sens d'un concept et à fixer l'usage recommandé pour des solutions de traduction. Il existe également des entrées normatives pour lesquelles la délimitation des contours de sens du concept et les solutions de traduction ont une valeur de norme, c'est-à-dire qu'elles excluent tout autre possibilité ou élargissement de sens.

53 L'objectif d'AGORA est de dépasser le stade de la terminologie bilingue classique (la mise en regard d'un terme en langue $A$ et d'un ou plusieurs termes correspondants en langue B) pour tendre vers une terminologie cognitive centrée sur des définitions d'experts, de vulgarisation ou encore des définitions tirées de textes normatifs (voir Raus, 2013, pour une étude des différentes démarches terminologiques existantes).

La terminologie d'AGORA présente un caractère à la fois descriptif et prescriptif, voire normatif dans certains cas précis. Cette dualité descriptif vs prescriptif/normatif reflète le double objectif d'AGORA: gérer la connaissance et proposer des solutions de traduction. Les champs de définition, les notes techniques décrivent le sens du concept exprimé par le mot source tandis que les termes cibles sont autant de propositions de solutions de traduction dont le caractère va du prescriptif au normatif dans des cas très précis. Ainsi, à un "balance sheet account » tel qu'il est utilisé dans le texte original anglais du Système de comptabilité nationale (ONU, 1993) correspondra un " compte de patrimoine » (terminologie normative), terme fixé non modifiable et non substituable par « compte de bilan » (terminologie prescriptive) comme c'est le cas dans les normes de comptabilité d'entreprise. La majorité des solutions de traduction proposées garde néanmoins un caractère purement prescriptif. Ainsi, la définition technique d'un terme comme « hedge fund» montre que la caractéristique commune de ces fonds n'est pas la pratique d'opérations spéculatives, mais d'opérations d'arbitrage (recherche à tout instant de la position financière la plus rentable). En l'occurrence, cette définition permet de remettre en cause la traduction par «fonds spéculatif » très utilisée par les journalistes et de préconiser la traduction par « fonds d'arbitrage ».

Cette cohabitation de types de terminologie différents s'explique également par la triple fonction de l'OCDE, organisme de réflexion, de prospective, mais aussi de normalisation dans de nombreux domaines. Dans tous les cas de figure, la présence de telles entrées s'inscrit dans une démarche cognitive - l'explicitation des connaissances de l'auteur et la description univoque des termes employés dans une norme.

En réunissant l'ensemble des travaux terminologiques menés dans le cadre de l'OCDE, AGORA met particulièrement en lumière cette diversité. Mais cette dernière n'est pas propre à l'OCDE. On la retrouve dans les travaux terminologiques de l'Organisation des Nations unies, du Fonds monétaire international ou de l'Union internationale des télécommunications, dans lesquelles il existe une tradition ancienne de terminologie traductionnelle de la part de leurs services linguistiques parallèlement à des réflexions terminologiques descriptives, prescriptives ou normatives (voir Raus, 2013, notamment les pages consacrées au débat sur la notion de « genre » dans de telles organisations).

57 Cette diversité reflète bien la diversité des thématiques traitées à l'OCDE, conformément à l'idée que les informations terminologiques doivent rendre "fidèlement compte d'un usage véritable, observé dans des textes écrits ou des transcriptions d'échanges oraux » 
(van Campenhoudt, 1999). Comme on vient de le voir, cette diversité est maitrisée au sens où le contenu terminologique de ce portail est prioritairement guidé par l'orientation et l'ergonomie résolument cognitives du projet, même si la finalité traductionnelle classique dans les outils terminologiques multilingues des organisations internationales reste largement présente dans AGORA.

Deux traits spécifiques d'AGORA témoignent du changement de paradigme intervenu entre le projet principalement traductionnel initial et le projet principalement cognitif actuel :

- même s'il demeure administré par la Division de la traduction de l'OCDE, le portail n'est pas celui de cette division, mais il constitue la base de connaissance de l'organisation dans son ensemble ;

- depuis le début de l'année 2016, un peu plus de 600 entrées ont été créées, mais surtout plus de 500 d'entre elles comportent des définitions émanant de l'OCDE.

De plus, AGORA s'appuie sur une base technologique flexible qui permet de diversifier les modalités de diffusion de son contenu sous des formes et des applications elles aussi diversifiées en fonction des publics visés et des besoins de communication des différentes directions de l'Organisation.

Enfin cette diversité des applications s'inscrit dans une tendance plus pragmatique et moins rigoureusement terminographique que certains chercheurs entendent promouvoir.

Admettre la pluralité en terminologie est déjà un pas, mais intégrer la diversité dans la théorie semble être le chemin pour faire de la terminologie une véritable branche des sciences du langage. (Desmet, $2002: 154)$

\section{Conclusion}

61 La démarche terminologique adoptée à l'OCDE s'inscrit naturellement dans une organisation dont l'activité est elle-même fondée sur la connaissance. Néanmoins, la problématique de la connaissance se pose pour la plupart des grandes organisations publiques et privées. Plus précisément, ce problème est exacerbé par le risque de la disparition de la connaissance liée à la mobilité grandissante des experts. Il est en effet rare qu'un expert quitte une organisation en léguant à ses collègues une forme quelconque de compendium de son savoir.

62 La terminologie telle qu'elle est pratiquée dans cette organisation constitue une réponse ergonomique à cette problématique aussi bien pour les experts que pour les traducteurs en tant que travailleurs de la connaissance. Mais il s'agit d'une réponse dans le cadre d'une ergonomie au sens plus large. C'est un moyen technologique de contribuer à l'enrichissement de la connaissance. Au niveau des traducteurs, c'est un formidable vecteur de formation spécialisée et notamment de formation continue. Cette problématique plus large qui relève de l'ergonomie générale de la traduction doit donner lieu à une réflexion des associations professionnelles et des formations pour penser le traducteur de demain. 


\section{BIBLIOGRAPHIE}

BESSÉ Bruno DE (1990), « La définition terminologique », Centre d’Études du Lexique (dir.), La

définition, Paris : Larousse, 252-260.

BLANCHON Élisabeth (1997), « Point de vue sur la définition », Meta, 42(1), 168-173, <id.erudit.org/ iderudit/002090ar>.

CHAMPSAUR Caroline et RochaRd Michel (2010), « Ergonomie et traduction : un exemple d'appropriation des outils de traduction », Traduire, 224, 7-13, Paris : Société française des traducteurs.

DelEDALLE Gérard (1995), La philosophie peut-elle être américaine ? Nationalité et universalité, Paris : Jacques Grancher.

DESMET Isabel (2002), « L'analyse du sens en terminologie : théorie et pratique de la définition terminologique », TradTerm, 8, 149-168, <www.revistas.usp.br/tradterm/article/ viewFile/49129/53209.pdf>.

DEWEY John (1986, $1^{\text {re }}$ éd. 1938), Logic, the Theory of Inquiry, The Later Works of John Dewey 1925-1953 (12), Carbondale, États-Unis : Southern Illinois University Press.

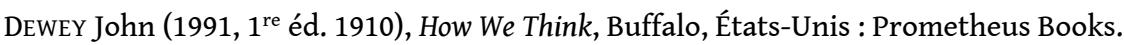

ÉCOLE NATIONALE SUPÉRIEURE DES SCIENCES DE L'INFORMATION ET DES BIBLIOTHÈQUES - ENSSIB (2012), « Article Bibliothèque numérique », <www.enssib.fr/le-dictionnaire/bibliotheques-numeriques>.

FOUGNER RYDNING Antin (2008), « La démarche de l'expert-traducteur face à un problème de reformulation », Meta, 53(4), 748-764, <http://www.erudit.org/revue/meta/2008/v53/ n4/019645ar.html>.

GouAdEc Daniel (1990), Terminologie. Constitution des données, Paris : Afnor, <www.gouadec.net/ publications/Terminologie_ConstitutionDonnees.pdf>.

Humbley John (1998), « Le terminologue et le spécialiste de domaine », ASp (revue du Groupe d'étude et de recherches en anglais de spécialité), 19-22, 137-150, <asp.revues.org/2789>.

HUMBLEY John (2011), « Terminologie et traduction, une complémentarité oubliée ?», communication présentée au colloque Tralogy, Paris, CNRS, <lodel.irevues.inist.fr/tralogy/ index.php?id=63>.

HUSTAD Eli \& BECHINA Aurilla Aurelie (2012), « Exploring the Role of Boundary Spanning in Distributed Networks of Knowledge », Electronic Journal of Knowledge Management, 10(2), < www.ejkm.com/issue/download.html ?idArticle =324>.

KIMBLE Chris (2013), « Knowledge Management, Codification and Tacit Knowledge », Information Research, 18(2), <www.informationr.net/ir/18-2/paper577.html\#.VjNqQ_kqnGg>.

JouRnAl OFFICIEL DE LA RéPUblique FRANÇAISE (1982), « Arrêté du 22 décembre 1981 », 624, Paris : JORF, <www.culture.fr/franceterme>.

LAVAULT-OlLÉON Élisabeth \& CARRÉ Alice (2012), «Traduction spécialisée : l'ergonomie cognitive au service de la formation ", ASp (62), 67-77, <asp.revues.org/3117>. 
LAVAULT-OLLÉON Élisabeth (2011), » L'ergonomie, nouveau paradigme pour la traductologie », ILCEA (14), <ilcea.revues.org/1031>.

ORGANISATION DE COOPÉRATION ET DE DÉVELOPPEMENT ÉCONOMIQUES - OCDE (2003), Manuel de Frascati : Méthode type proposée pour les enquêtes sur la recherche et le développement expérimental (6 éd.), Paris : Éditions de l'OCDE.

ORGANISATION DE COOPÉRATION ET DE DÉVELOPPEMENT ÉCONOMIQUES - OCDE (2014), Modèle de convention fiscale de l'OCDE concernant le revenu et la fortune (version abrégée), Paris : Éditions de l'OCDE.

ORGANISATION DE COOPÉRATION ET DE DÉVELOPPEMENT ÉCONOMIQUES - OCDE, COMMISSION EUROPÉENNE (2005), Manuel d'Oslo: Principes directeurs pour le recueil et l'interprétation des données sur l'innovation ( $3^{\mathrm{e}}$ éd.), Paris : Éditions de l'OCDE.

ORGANISATION DES NATIONS UNIES - ONU, FONDS MONÉTAIRE INTERNATIONAL - FMI, BANQUE MONDIALE, E UROSTAT, ORGANISATION DE COOPÉRATION ET DE DÉVELOPPEMENT ÉCONOMIQUES - OCDE (1993), Système de comptabilité nationale 1993 (SCN93), titre original : System of National Accounts 1993, New York, ÉtatsUnis : ONU.

RAuS Rachele (2013), La terminologie multilingue : la traduction des termes de l'égalité homme/femme dans le discours international, Bruxelles : De Boeck.

ROCHARD Michel (1992), « Le traducteur face à la terminologie : consommateur ou acteur ? ", Terminologies et Traduction (2-3), 105-112, Luxembourg : Office des publications officielles des Communautés européennes.

ROCHARD Michel (2006), « La rétroconception entre compréhension et ré-expression des textes économiques ", E. Lavault-Olléon (dir.), Traduction spécialisée : pratiques, théories, formations, Berne : Peter Lang, 103-113.

RocHE Christophe (à paraître), «Une Théorie du Concept pour la Terminologie (CTT : a Concept Theory of Terminology)», The Definition in Terminology, Berlin : Frank \& Timme Publisher.

SELESKOVITCH Danica \& LEDERER Marianne (1989), Pédagogie raisonnée de l'interprétation, Paris : Didier Érudition.

VAN CAMPENHOUDT Marc (1999), « Terminologie descriptive : petite initiation à l'exploitation de corpus ", communication présentée dans le cadre de la $8^{\mathrm{e}}$ Université d'automne en terminologie, Université Rennes 2, en ligne sur <termisti.ulb.ac.be/rennes99.pdf>.

\section{NOTES}

1. On notera que c'est la même préoccupation d'efficience (ne pas retraduire ce qu'on a déjà traduit) qui fait le succès des mémoires de traduction.

2. Malgré toute la bonne volonté dont font preuve les experts à l'égard des traducteurs.

3. La distinction entre traducteurs et interprètes selon le critère du temps dont ils disposent respectivement est à relativiser dans la pratique moderne.

4. Un prix de transfert est un prix, adopté à des fins comptables, qui est utilisé pour valoriser les opérations entre entreprises affiliées intégrées sous la même direction à un niveau artificiellement haut ou bas pour payer des revenus ou effectuer des transferts en capital non déclarés entre ces entreprises. (SCN93, §3, 1979)

5. Définition extraite du portail terminologique AGORA de l'OCDE. Cette définition d'expert illustre bien le risque de perte de connaissance dans des organisations. La page Web où figurait ce 
glossaire a en effet disparu du site Web de l'OCDE. Seule la consignation de cette définition dans le portail a permis d'en conserver la trace.

6. Définition extraite du portail terminologique AGORA de l'OCDE.

7. Extrait du champ de précision sur la traduction de l'entrée capital loss du portail terminologique AGORA de l'OCDE.

\section{RÉSUMÉS}

Le traducteur spécialisé et l'auteur-expert ont un rapport différent au monde et aux mots. Dans ses travaux, l'expert choisit des mots pour décrire une réalité complexe alors que le traducteur a besoin de comprendre quel concept ou découpage du monde correspond aux mots de l'expert. Les deux démarches sont complémentaires, l'expert et le traducteur étant tous deux des «travailleurs de la connaissance ». Internet est aujourd'hui un outil irremplaçable pour l'expert, comme pour le traducteur. Mais pour accroître l'impact de ses analyses et de ses réflexions, l'expert ne doit pas se contenter de partager ses analyses. Il doit également fournir les clefs nécessaires à la compréhension de ce travail, il doit partager son savoir. Le travail terminologique du traducteur peut l'y aider.

Le développement d'une culture numérique du partage (multiplication des réseaux sociaux comme outils de communication de masse) a vu naitre de nouvelles formes de collaboration. Le portail terminologique collaboratif semble être la plateforme idéale pour répondre à la fois aux besoins de l'expert (faire connaitre son travail et en améliorer l'impact) et à ceux du traducteur (utiliser et conserver des définitions fiables). Nous montrerons comment, en rassemblant les données de multiples spécialités et en plaçant le concept (terme et définition) au centre de l'entrée terminologique, le portail collaboratif met les solutions ergonomiques du numérique au service d'une véritable ergonomie cognitive.

The specialised translator and the author-expert have a different relationship with both the world and words. In their work, experts choose words to describe a complex reality whereas translators need to understand the concept or worldview reflected in the experts' words. The two approaches are complementary, as both the expert and the translator are "knowledge workers". These days, the Internet is an indispensable tool for both experts and translators. But simply sharing findings is not enough for experts seeking to increase the impact of their reflections and analyses. They must also provide the keys necessary to understand their work and share their knowledge. Terminology work by translators can help them do this.

The development of a culture of digital sharing (with the proliferation of mass-media social networks) has given rise to new types of collaboration. The collaborative terminology portal seems to be the ideal platform for addressing the needs of both experts (promoting and enhancing the impact of their work) and translators (the use and conservation of reliable definitions). This paper will show how, by aggregating data on a multitude of specialised subjects and focusing terminological input on the concept (term and definition), the collaborative portal can develop genuine cognitive ergonomics by leveraging the ergonomic solutions provided by digital technology. 
INDEX

Mots-clés : connaissance, ergonomie, portail collaboratif, terminologie

Keywords : knowledge, ergonomics collaborative portal, terminology

\section{AUTEURS}

CAROLINE CHAMPSAUR

OCDE, Paris

MICHEL ROCHARD

OCDE, Paris 